PROCEEDINGS OF THE

AMERICAN MATHEMATICAL SOCIETY

Volume 140, Number 5, May 2012, Pages 1473-1481

S 0002-9939(2011)11153-5

Article electronically published on August 18, 2011

\title{
HEREDITARY ORDERS IN THE QUOTIENT RING OF A SKEW POLYNOMIAL RING
}

\author{
JOHN S. KAUTA
}

(Communicated by Birge Huisgen-Zimmermann)

\begin{abstract}
Let $K$ be a field, and let $\sigma$ be an automorphism of $K$ of finite order. Let $K(X ; \sigma)$ be the quotient ring of the skew polynomial ring $K[X ; \sigma]$. Then any order in $K(X ; \sigma)$ which contains $K$ and its center is a valuation ring of the center of $K(X ; \sigma)$ is a crossed-product algebra $A_{f}$, where $f$ is some normalized 2-cocycle. Associated to $f$ is a subgroup $H$ of $\langle\sigma\rangle$ and a graph. In this paper, we determine the connections between hereditary-ness and maximal order properties of $A_{f}$ and the properties of $H, f$ and the graph of $f$.
\end{abstract}

\section{INTRODUCTION}

If $R$ is a ring, then $J(R)$ will denote its Jacobson radical, $Z(R)$ its center, $U(R)$ its group of multiplicative units, and $R^{\#}$ the subset of all the non-zero elements. The ring $R$ is called hereditary if all left ideals are projective as left $R$-modules and all right ideals are projective as right $R$-modules. It is called Bézout if finitely generated one-sided ideals are principal.

Let $V$ be a valuation ring of a field $F$. If $Q$ is a finite-dimensional central simple $F$-algebra, then a subring $R$ of $Q$ is called an order in $Q$ if $R F=Q$. If in addition $V \subseteq R$ and $R$ is integral over $V$, then $R$ is called a $V$-order. If a $V$-order $R$ is maximal among the $V$-orders of $Q$ with respect to inclusion, then $R$ is called a maximal $V$-order (or just a maximal order if the context is clear). Examples of maximal orders are Azumaya algebras over a valuation ring. An order $R$ in $Q$ is called a valuation ring of $Q$ if it is Bézout and $R / J(R)$ is a simple Artinian ring. If $V$ is a DVR, then a subring of $Q$ with center $V$ is a valuation ring if and only if it is a maximal order (see [6, Example 1.15]). Now assume $Q$ is a division ring. Then a subring $R$ of $Q$ is called a total valuation ring of $Q$ if, given any $0 \neq \alpha \in Q$, either $\alpha \in R$ or $\alpha^{-1} \in R$. A total valuation $\operatorname{ring} R$ of $Q$ is called an invariant valuation ring of $Q$ if it is invariant under inner automorphisms of $Q$. A valuation $\operatorname{ring} R$ of a finite dimensional division ring is a total valuation ring if and only if $R / J(R)$ is a division algebra by [6, Theorem G]. When $V$ is a DVR, the class of total valuation rings of $Q$ with center $V$ coincides with the class of invariant valuation rings of $Q$ with center $V$. For further information about properties of such rings, the reader is referred to [6].

For the moment, consider the following setup: $V$ a DVR, with quotient field $F$; $K / F$ a finite Galois extension, with group $G ; S$ the integral closure of $V$ in $K$;

Received by the editors September 21, 2009 and, in revised form, January 8, 2011.

2010 Mathematics Subject Classification. Primary 16S35, 16S36, 16E60; Secondary 13F30.

(C)2011 American Mathematical Society 
$f: G \times G \mapsto S^{\#}$ a normalized cocycle, i.e., a function satisfying $f^{\sigma}(\tau, \gamma) f(\sigma, \tau \gamma)=$ $f(\sigma, \tau) f(\sigma \tau, \gamma)$ for all $\sigma, \tau, \gamma \in G$ and $f(1, \sigma)=f(\sigma, 1)=1$ for all $\sigma \in G$. One can construct a "crossed-product" $V$-algebra

$$
A_{f}=\sum_{\sigma \in G} S x_{\sigma}
$$

with the usual rules of multiplication $\left(x_{\sigma} s=\sigma(s) x_{\sigma}\right.$ for all $s \in S, \sigma \in G$ and $\left.x_{\sigma} x_{\tau}=f(\sigma, \tau) x_{\sigma \tau}\right)$.

Then $A_{f}$ is associative, with identity $1=x_{1}$, and center $V=V x_{1}$. Further, $A_{f}$ is a $V$-order in the crossed product $F$-algebra $Q_{f}=\sum_{\sigma \in G} K x_{\sigma}$. Two such cocycles $f$ and $g$ are said to be cohomologous over $S$ (respectively cohomologous over $K$ ), and we write $f \sim_{S} g$ (respectively $f \sim_{K} g$ ) if there are elements $\left\{c_{\sigma} \mid \sigma \in G\right\} \subseteq U(S)$ (respectively $\left.\left\{c_{\sigma} \mid \sigma \in G\right\} \subseteq U(K)\right)$ such that $g(\sigma, \tau)=c_{\sigma} \sigma\left(c_{\tau}\right) c_{\sigma \tau}^{-1} f(\sigma, \tau)$ for all $\sigma, \tau \in G$. Let $H_{f}=\left\{\sigma \in G \mid f\left(\sigma, \sigma^{-1}\right) \in U(S)\right\}$. Then $H_{f}$ is a subgroup of $G$ which we will sometimes denote simply by $H$ if the context is clear. On $G / H$, the left coset space of $G$ by $H$, one can define a partial ordering by the rule

$$
\sigma H \leq \tau H \text { if } f\left(\sigma, \sigma^{-1} \tau\right) \in U(S) .
$$

Then " $\leq$ " is well-defined and depends only on the cohomology class of $f$ over $S$. Further, $H$ is the unique least element. We call this partial ordering on $G / H$ the graph of $f$.

Such a setup was first formulated by Haile in [3, with the assumption that $S$ is unramified over $V$. Haile [3] did not deal with the case when $H=G$ in his paper, but that is hardly an impediment to the beautiful theory he developed: When $H=G$, then $A_{f}$ is a classical crossed product algebra. It is Azumaya over $V$ since $S / V$ is a Galois extension of rings.

Now suppose $H \neq G$. If $A_{f}$ is Azumaya over $V$, then $J\left(A_{f}\right)=J(V) A_{f}$; hence, by [3. Proposition 3.1(b)], we infer that for each $\sigma \in G, f\left(\sigma, \sigma^{-1}\right) \notin M$ for any maximal ideal $M$ of $S$. This implies that $H=G$, which is a contradiction.

Thus we have:

Sublemma. With the notation as above and assuming $S$ is unramified over $V$, $H=G$ if and only if $A_{f}$ is an Azumaya algebra.

Here is another minor fact: if $H=G$, then $A_{f}$ is obviously primary. Therefore by [3, Proposition 2.1], if $S$ is a valuation ring, then $A_{f}$ is always primary whether or not $H=G$.

Let us now fix some notation to be used in the rest of the paper. Let $K$ denote a field, and let $\sigma$ be an automorphism of $K$ of finite order $n$. To avoid trivialities, we will assume $n>1$. Consider the skew polynomial ring $K[X ; \sigma]$ over $K$ with multiplication defined by the commutation rule: $X a=\sigma(a) X$ for all $a \in K$. Let $K(X ; \sigma)$ denote the quotient ring of $K[X ; \sigma]$. Then $K(X ; \sigma)$ is a finite dimensional division algebra.

It is known that $K(X ; \sigma)$ is a cyclic algebra; e.g., see [1, $\S 12.2$, Example 5]. For the convenience of the reader, we shall outline a proof of this fact here: Let $F$ be the fixed field of $\sigma$. Then $X^{n} \in Z(K(X ; \sigma))$, and $K\left(X^{n}\right)$ is a Galois extension of $F\left(X^{n}\right)$ with group $G:=\langle\sigma\rangle$. If $\left\langle K\left(X^{n}\right), X\right\rangle$ is the subalgebra of $K(X ; \sigma)$ generated by $K\left(X^{n}\right)$ and $X$, then $\left\langle K\left(X^{n}\right), X\right\rangle \cong\left(K\left(X^{n}\right) / F\left(X^{n}\right), \sigma, X^{n}\right)$, a cyclic $F\left(X^{n}\right)$ algebra. Since $K[X ; \sigma] \subseteq\left\langle K\left(X^{n}\right), X\right\rangle \subseteq K(X ; \sigma)$, we conclude that $\left\langle K\left(X^{n}\right), X\right\rangle=$ $K(X ; \sigma)$. 
In $K(X ; \sigma)$, we also have the subring $K\left[X^{-1} ; \sigma\right]$ of all polynomials in $X^{-1}$ with coefficients in $K$ with multiplication: $X^{-1} a=\sigma^{-1}(a) X^{-1}$. Its quotient ring is also $K(X ; \sigma)$ and $\left\langle K\left(X^{n}\right), X^{-1}\right\rangle=K(X ; \sigma)$.

We will therefore identify $K(X ; \sigma)$ with either

$$
Q_{f_{1}}=\left(K\left(X^{n}\right) / F\left(X^{n}\right), \sigma, X^{n}\right) \text { or } Q_{f_{2}}=\left(K\left(X^{n}\right) / F\left(X^{n}\right), \sigma^{-1}, X^{-n}\right),
$$

where for $1 \leq i, j<n, f_{1}\left(\sigma^{i}, \sigma^{j}\right)=f_{2}\left(\sigma^{-i}, \sigma^{-j}\right)=1$ if $i+j<n$; otherwise $f_{1}\left(\sigma^{i}, \sigma^{j}\right)=X^{n}$ and $f_{2}\left(\sigma^{-i}, \sigma^{-j}\right)=X^{-n}$. We have $f_{1} \sim_{K} f_{2}$.

Let $V$ be a valuation ring of $F\left(X^{n}\right)$ that contains $F$. Then $V \supseteq F\left[X^{n}\right]$ or $V \supseteq F\left[X^{-n}\right]$. Whatever is the case, $V$ is a DVR of $F\left(X^{n}\right)$. Let $S$ be the integral closure of $V$ in $K\left(X^{n}\right)$. Then $S=K V$. Since $K$ is Galois over $F$, there is an element $t \in K$ of trace 1 . Since $t \in S$, we see that $K\left(X^{n}\right) / F\left(X^{n}\right)$ is a tamely ramified extension. We will now see that it is actually an unramified extension.

Let $W_{1}, \ldots, W_{r}$ be all the extensions of $V$ to $K\left(X^{n}\right)$. Assume $V \supseteq F\left[X^{n}\right]$. Then $J\left(W_{i}\right)=q_{i} W_{i}$, where $q_{i}$ is a monic irreducible polynomial in $K\left[X^{n}\right]$. Given a $\tau \in G$ then, in the ring $K\left[X^{n}\right], \tau\left(q_{i}\right)$ is an associate of $q_{j}$ for some $j$. But since the $q_{k}$ as well as the $\tau\left(q_{k}\right)$ are monic, we actually have equality: $\tau\left(q_{i}\right)=q_{j}$ for some $j$. Thus $G$ permutes the $q_{k}$, and therefore $p=q_{1} q_{2} \cdots q_{r}$ is fixed by $G$, and hence is an element of $F\left[X^{n}\right]$. We conclude that the ramification index of $S$ over $V$ is 1 . The same conclusion holds when $V \supseteq F\left[X^{-n}\right]$. Hence $K\left(X^{n}\right) / F\left(X^{n}\right)$ is an unramified extension.

Now let $A$ be an order in $K(X ; \sigma)$ containing $K$ whose center is a valuation ring $V$. Then $V$ must contain $F$ and is therefore a DVR. Such orders are automatically $V$-orders. Moreover, in this case, $A \supseteq K V=S$. Since $S$ is unramified over $V$, by [3, Proposition 1.3] we have

$$
A=A_{f}=\sum_{i=o}^{n-1} S x_{\sigma^{i}},
$$

a "crossed-product" $V$-algebra, where $x_{\sigma^{i}}=k_{i} X^{i}\left(x_{\sigma^{i}}=k_{i} X^{-i}\right.$ if $\left.X^{n} \notin V\right)$, $1=k_{0}, k_{1}, \ldots, k_{n-1} \in K\left(X^{n}\right)^{\#}$, and $f: G \times G \mapsto S^{\#}$ is a normalized 2-cocycle cohomologous to $f_{1}$ (as well as $f_{2}$ ) over $K$.

Let $A_{f}$ and $A_{g}$ be two $V$-orders in $K(X ; \sigma)$ containing $K$. Then $f \sim_{S} g$ if and only if there exists a $V$-algebra isomorphism $\phi: A_{f} \mapsto A_{g}$ such that $\phi(s)=s$ for all $s \in S$. Suppose $f \sim_{S} g$ and $\phi$ is such an isomorphism. Then there exists a $q=c_{0}+c_{1} X+c_{2} X^{2}+\cdots+c_{m} X^{m} \in K[X ; \sigma]^{\#}$ such that $\phi$ is conjugation by $q$. Since $K \subseteq S, \phi(t)=t$ for all $t \in K$ and hence $q t=t q$ for all $t \in K$. Thus, for each $i, c_{i} \sigma^{i}(t)=t c_{i}$ for all $t \in K$. So $c_{i}=0$ if $i \neq 0$; hence $q=c_{0} \in K^{\#} \subseteq U\left(A_{f}\right)$. Hence $A_{g}=\phi\left(A_{f}\right)=c_{0} A_{f} c_{0}^{-1}=A_{f}$. Conversely, if $A_{f}=A_{g}$, then $f \sim_{S} g$.

We therefore have the following lemma:

Lemma 1.1. (1) Let $V$ be a valuation ring of $F\left(X^{n}\right)$ that contains $F$, and let $S$ be the integral closure of $V$ in $K\left(X^{n}\right)$. Then $V$ is a DVR and $S$ is unramified over $V$.

(2) If $A$ is an order in $K(X ; \sigma)$ containing $K$ and whose center is a valuation ring, then the center is a DVR as in part (1) above and $A=A_{f}$, a crossed-product order, where $f: G \times G \mapsto S^{\#}$ is a normalized 2-cocycle cohomologous to $f_{1}$ and $f_{2}$ over $K$. 
(3) If $A_{f}$ and $A_{g}$ are two such orders of $K(X ; \sigma)$, then $f \sim_{S} g$ if and only if $A_{f}=A_{g}$. That is, if $\left\{A_{f}\right\}$ are orders in $K(X ; \sigma)$ of this type, then there is a one-to-one correspondence between such orders and cohomology classes of $f$ over $S$.

For the rest of this paper, $V$ will denote a valuation ring of $F\left(X^{n}\right)$ that contains $F$, and $S$ will denote its integral closure in $K\left(X^{n}\right)$. Either $X^{n} \in V$ or $X^{-n} \in V$ (or both). If $X^{n} \in V$, we will let

$$
A_{f_{1}}=\sum_{i=o}^{n-1} S X^{i}
$$

and if $X^{-n} \in V$, we will let

$$
A_{f_{2}}=\sum_{i=o}^{n-1} S X^{-i}
$$

These are crossed-product $V$-orders in $K(X ; \sigma)$ with respect to the cocycles $f_{1}$ and $f_{2}$ described above. Observe that if $X^{n} \in U(V)$, then $A_{f_{2}}=X^{n} A_{f_{2}}=A_{f_{1}}$ and $f_{1} \sim_{S} f_{2}$.

In this paper we want to give an explicit description, in terms of crossed-product orders 11 of all hereditary and maximal orders in $K(X ; \sigma)$ that contain $K$ and whose center is a valuation ring. In so doing, we will determine the connections between hereditary-ness and maximal order properties of $A_{f}$, and the properties of $H, f$, and the graph of $f$. Our main result is Theorem 3.2.

\section{MaXimal orders in $K(X ; \sigma)$ CONTAining $K$}

Let $A$ be a maximal $V$-order in $K(X ; \sigma)$ containing $K$. Then $A=A_{f}$ for some normalized 2-cocycle $f: G \times G \mapsto S^{\#}$ by Lemma 1.1. We consider three cases:

Case 1: $X^{n} \in V$, but $X^{-n} \notin V$. We know that $V=F\left[X^{n}\right]_{P}$ for some maximal ideal $P$ of $F\left[X^{n}\right]$. Since $X^{n} \notin U(V), X^{n} \in P$; hence $X^{n} F\left[X^{n}\right] \subseteq P$, forcing $P=X^{n} F\left[X^{n}\right]$. Thus $V=F\left[X^{n}\right]_{X^{n} F\left[X^{n}\right]}$ and $S=K\left[X^{n}\right]_{X^{n} K\left[X^{n}\right]}$, which is a DVR unramified over $V$. In this case, $H_{f_{1}}=\{1\}, f_{1}\left(\sigma, \sigma^{-1}\right)=X^{n} \in X^{n} S \backslash X^{2 n} S$, and the graph of $f_{1}$ is the simple chain

$$
H_{f_{1}} \leq \sigma H_{f_{1}} \leq \sigma^{2} H_{f_{1}} \leq \cdots \leq \sigma^{n-1} H_{f_{1}} .
$$

Hence $A_{f_{1}}$ is a maximal order by [3, Theorem 2.3].

It is well known that $K[X ; \sigma]$ is a Bézout ring, since $K$ is a field and $\sigma$ is an automorphism of $K$. Thus $K[X ; \sigma]$ is a prime PI Bézout ring; hence, by [2, Theorem 2.6], it is localizable at the maximal ideal $X K[X ; \sigma]$, and if $B$ is the localization of $K[X ; \sigma]$ at $X K[X ; \sigma]$, then $B$ is a valuation ring of $K(X ; \sigma)$. Observe that $B / J(B)=K$, a field; therefore $B$ is a total valuation ring. Since $Z(B)=V$ is a DVR, $B$ must be an invariant valuation ring of $K(X ; \sigma)$. Thus $A_{f}=B=A_{f_{1}}$. By the sublemma, $A_{f}$ is not Azumaya over $V$.

Case 2: $X^{-n} \in V$, but $X^{n} \notin V$. This is a mirror image of Case 1 . We readily see that $A_{f}=A_{f_{2}}$. It is an invariant valuation ring of $K(X ; \sigma)$, but not an Azumaya algebra.

Case 3: $X^{n} \in U(V)$. Since $S / V$ is a Galois extension of rings and $f_{1}: G \times G \mapsto$ $U(S), A_{f_{1}}$ is Azumaya over $V$. We therefore have $A_{f} \cong A_{f_{1}}=A_{f_{2}}$.

\footnotetext{
${ }^{1}$ Note that since $f$ does not necessarily take values in $U(S), A_{f}$ may not be a classical crossedproduct algebra. This is important to bear in mind throughout this paper.
} 
Therefore we have the following theorem:

Theorem 2.1. Let $A$ be a $V$-order in $K(X ; \sigma)$ containing $K$ whose center is a valuation ring $V$. If $A$ is a maximal order, then:

(1) $A \cong A_{f_{1}}$ or $A \cong A_{f_{2}}$.

(2) $A$ is either an invariant valuation ring of $K(X ; \sigma)$ or an Azumaya algebra over its center or both.

(3) If $X^{n} \in V \backslash U(V)$ (respectively $X^{-n} \in V \backslash U(V)$ ), then $A=A_{f_{1}}$ (respectively $\left.A=A_{f_{2}}\right)$, and it is an invariant valuation ring of $K(X ; \sigma)$.

(4) $A$ is Azumaya over its center if and only if $X^{n}$ is a unit in $A$. When this occurs, $A_{f_{1}}=A_{f_{2}}$.

Conversely, if $A \cong A_{f_{1}}$ or $A \cong A_{f_{2}}$, then $A$ is a maximal order.

Remark 2.2. A characterization of maximal $V$-orders in $K(X ; \sigma)$ in terms of $H$, $f$, and the graph of $f$ is a straightforward application of the sublemma and [3, Theorem 2.3]. The order $A_{f}$ is maximal if and only if one of the following is true:

(1) the graph of $f$ is trivial;

(2) $H=\{1\}$, the graph of $f$ is $H \leq \sigma H \leq \sigma^{2} H \leq \cdots \leq \sigma^{n-1} H$, and $f\left(\sigma, \sigma^{-1}\right) \in J(S) \backslash J(S)^{2}$

(3) or $H=\{1\}$, the graph of $f$ is $H \leq \sigma^{-1} H \leq \sigma^{-2} H \leq \cdots \leq \sigma^{1-n} H$, and $f\left(\sigma^{-1}, \sigma\right) \in J(S) \backslash J(S)^{2}$,

according to whether $X^{n} \in U(V), X^{n} \in V \backslash U(V)$, or $X^{-n} \in V \backslash U(V)$.

We now want to determine the number of maximal $V$-orders in $K(X ; \sigma)$ that contain $K$.

Suppose $V$ is indecomposed in $K\left(X^{n}\right)$. If $X^{n} \notin V$ or $x^{-n} \notin V$, then by Theorem 2.1 there is only one maximal $V$-order in $K(X ; \sigma)$ and it is an invariant valuation ring and we are done. On the other hand, if $X^{n} \in U(V)$, then $A_{f_{1}}$ is an Azumaya algebra by Theorem 2.1. Let $B$ be a maximal $V$-order in $K(X ; \sigma)$ containing $K$. Then, since $B \supseteq S$, we have $B=A_{g}$ for some 2-cocycle $g: G \times G \mapsto S^{\#}$. In fact, the proof of [3, Proposition 1.3] shows that there exists $1=k_{0}, k_{1}, \ldots, k_{n-1} \in K\left(X^{n}\right)^{\#}$ such that

$$
A_{g}=\sum_{i=o}^{n-1} S k_{i} X^{i} .
$$

So if $i+j<n$, then $\left(k_{i} X^{i}\right)\left(k_{j} X^{j}\right)=g\left(\sigma^{i}, \sigma^{j}\right) k_{i+j} X^{i+j}$ and $g\left(\sigma^{i}, \sigma^{j}\right)=k_{i} \sigma^{i}\left(k_{j}\right) /$ $k_{i+j}$. All maximal $V$-orders in $K(X ; \sigma)$ are conjugate, because $V$ is a DVR; hence $B$ must be an Azumaya algebra as well, and so by the sublemma we have $g\left(\sigma^{i}, \sigma^{j}\right) \in$ $U(S)$ for all $i, j$. Let $v$ be a valuation on $K\left(X^{n}\right)$ corresponding to the valuation ring $S$. Since $v\left(g\left(\sigma^{i}, \sigma^{j}\right)\right)=0$ for all $i, j$, we have $v\left(k_{i+j}\right)=v\left(k_{i}\right)+v\left(k_{j}\right)$ if $i+j<n$; therefore

$$
v\left(k_{i}\right)=i v\left(k_{1}\right), \quad 0 \leq i<n .
$$

If $v\left(k_{1}\right)>0$, then $v\left(k_{i}\right)>0$ for all $1 \leq i<n$; hence

$$
B=\sum_{i=o}^{n-1} S k_{i} X^{i} \varsubsetneqq \sum_{i=o}^{n-1} S X^{i}=A_{f_{1}},
$$

a contradiction. If $v\left(k_{1}\right)<0$, then $B \supsetneqq A_{f_{1}}$, another contradiction. So $v\left(k_{i}\right)=0$ for all $i$; hence we must have $B=A_{f_{1}}$. Thus $A_{f_{1}}$ is the only maximal $V$-order of $K(X ; \sigma)$ containing $K$. 
Now suppose $V$ decomposes in $K\left(X^{n}\right)$. Let $B$ be a maximal $V$-order containing $K$. Then $B$ is not an invariant valuation ring; otherwise $S$ would be a valuation ring of $K\left(X^{n}\right)$. By Theorem 2.1,B is an Azumaya algebra over $V$ and $K\left[X^{n}\right] \subseteq$ $B$. If $J(V)=p V$, where $p=p\left(X^{n}\right)$ is an irreducible polynomial in $F\left[X^{n}\right]$, let $p=q_{1} q_{2} \cdots q_{r}$ be the complete factorization of $p$ in $K\left[X^{n}\right]$. Then $r>1$, since $V$ decomposes in $K\left(X^{n}\right)$, and the $q_{k}$ are distinct irreducible polynomials in $K\left[X^{n}\right]$, as in the proof of Lemma 1.1. Hence $q_{1}^{l} \notin p K\left[X^{n}\right]$ for any natural number $l$. Since $J(B)=p B$, we have, for any natural number $l$,

$$
q_{1}^{l} \in(B \backslash J(B)) \backslash U(B) .
$$

By [6, Lemma 3.2], $q_{1}^{l} B q_{1}^{-l} \neq B$ for all $l$. Since $q_{1}^{l} K q_{1}^{-l}=K$, we see that $B$ has infinitely many conjugates containing $K$.

We therefore have the following result:

Theorem 2.3. If $V$ is indecomposed in $K\left(X^{n}\right)$, then there is a unique maximal $V$-order in $K(X ; \sigma)$ containing $K$, namely $A_{f_{1}}$ or $A_{f_{2}}$. If $V$ is decomposed, then there are infinitely many maximal $V$-orders in $K(X ; \sigma)$ containing $K$. Moreover, $X^{n} \in U(V)$, and these maximal orders are Azumaya.

Remark 2.4. Even when $V$ is indecomposed in $K\left(X^{n}\right)$, if the maximal $V$-order containing $K$ is not an invariant valuation ring, then, by 6 , Theorem $\mathrm{G}$ and Corollary $\mathrm{G}]$, there are infinitely many maximal $V$-orders in $K(X ; \sigma)$, but the rest do not contain $K$.

\section{Hereditary orders in $K(X ; \sigma)$ Containing $K$}

We now want to determine the precise conditions for a $V$-order in $K(X ; \sigma)$ containing $K$ to be hereditary. But first, we need the following lemma, based on an analysis of [4, Definition 2.1(iii)] and that of the proof of [4, Theorem 2.4]. For purposes of this lemma, we need the following setup. Let $D$ be a finite dimensional division ring with center $L$, and let $U$ be a DVR of $L$. Let $\Delta$ be an invariant valuation ring of $D$ with center $U$, and let $v$ be a valuation on $D$ corresponding to $\Delta$ with value group $\mathbb{Z}$. For a fixed positive integer $k$, let $Q=M_{k}(D)$, a full matrix ring over $D$. If a $U$-order $A$ in $Q$ contains all the standard idempotents of $Q$, then $A=M_{k}\left(\Delta_{i j}\right)$, where each $\Delta_{i j}$ is a $U$-submodule of $D$. Assume that $\Delta_{i i}=\Delta$ for each $i$. Then each $\Delta_{i j}$ is a $\Delta$-submodule of $D$, since $R$ is a ring. We know there exists a $\delta \in \Delta$ such that $J(\Delta)=\delta \Delta$. Fix integers $i$ and $j$, with $1 \leq i, j \leq k$. Then there are fixed integers $a$ and $b$ such that $\Delta_{i j}=\delta^{a} \Delta$ and $\Delta_{j i}=\delta^{b} \Delta$.

Suppose $a+b>1$. Then $\alpha=\delta^{a-1}$ has the property that $\alpha \notin \delta^{a} \Delta=\Delta_{i j}$, and, since $1-a<b$, we also have $\alpha^{-1} \notin \delta^{b} \Delta=\Delta_{j i}$.

On the other hand, suppose $a+b \leq 1$. Let $0 \neq \alpha \in D$. If $\alpha \notin \Delta_{i j}$ and $\alpha^{-1} \notin \Delta_{j i}$, then $v(\alpha)<a$ and $v\left(\alpha^{-1}\right)<b$, and so $0=v\left(\alpha \cdot \alpha^{-1}\right)=v(\alpha)+v\left(\alpha^{-1}\right)<$ $a+v\left(\alpha^{-1}\right)<a+b \leq 1$; hence $0<a+v\left(\alpha^{-1}\right)<1$, a contradiction.

Thus for condition [4, Definition 2.1(iii)] to hold in our situation, the precise requirement is that $a+b \leq 1$; that is, we need to have $\delta^{a} \Delta \delta^{b} \Delta=\delta^{a+b} \Delta \supseteq J(\Delta)$.

We thus obtain the following corollary of [4, Theorem 2.4], valid since $U$ is a DVR:

Lemma 3.1. With the notation as above, $A$ is hereditary if and only if, for all $i$, $j, 1 \leq i, j \leq k$, we have $\Delta_{i j} \Delta_{j i} \supseteq J(\Delta)$. 
Let $A$ be an order in $K(X ; \sigma)$ containing $K$ whose center is a valuation ring $V$. If $A$ is hereditary, then it is well known that it is an intersection of maximal $V$-orders; e.g., see [5, Theorem 40.10]. If there exists only one maximal $V$-order containing $K$, then $A$ must be a maximal $V$-order.

From now on, assume there is more than one maximal $V$-order containing $K$. Therefore, by Theorem 2.3, $X^{n} \in U(V)$ and $V$ decomposes in $K\left(X^{n}\right)$. We know that

$$
A=A_{f}=\sum_{i=o}^{n-1} S x_{\sigma^{i}}
$$

where $x_{\sigma^{i}}=k_{i} X^{i}, 1=k_{0}, k_{1}, \ldots, k_{n-1} \in K\left(X^{n}\right)^{\#}$.

Note that, as $M$ varies over the set of maximal ideals of $S$, the corresponding decomposition groups coincide, since $G$ is an abelian group. We will denote this group by $G^{Z}$.

Let $M=M_{1}, M_{2}, \ldots, M_{r}$ be a complete list of maximal ideals of $S$. Let $F_{i}$ : $G^{Z} \times G^{Z} \mapsto S_{M_{i}}^{\#}$ be the restriction of $f$. If $\left(\widehat{F\left(X^{n}\right)}, \widehat{V}\right)$ is the completion of $\left(F\left(X^{n}\right), V\right)$, then

$$
S \otimes_{V} \widehat{V} \cong S_{1} \oplus \cdots \oplus S_{r} \text { and } K\left(X^{n}\right) \otimes_{F\left(X^{n}\right)} \widehat{F\left(X^{n}\right)} \cong K_{1} \oplus \cdots \oplus K_{r}
$$

where $\left(K_{i}, S_{i}\right)$ is the completion of $\left(K\left(X^{n}\right), S_{M_{i}}\right)$. Let $e_{i i}$ be the multiplicative identity of $S_{i}$. Let $g_{1}, g_{2}, \ldots, g_{r}$ be coset representatives of $G^{Z}$ in $G$ with $g_{i}\left(e_{11}\right)=$ $e_{i i}$. It is known that

$$
K(X ; \sigma) \otimes_{F\left(X^{n}\right)} \widehat{F\left(X^{n}\right)} \cong M_{r}\left(Q_{\hat{f}_{1}}\right)
$$

a full matrix ring over $Q_{\hat{f}_{1}}$, where $\hat{f}_{i}: G^{Z} \times G^{Z} \mapsto S_{i}^{\#}$ is the obvious "completion" of $F_{i}$ and $Q_{\hat{f}_{i}}:=\sum_{\sigma^{j} \in G^{z}} K_{i} X^{j} 2$ The $e_{i i}$ correspond to the standard orthogonal idempotents of the matrix ring above. Observe that $e_{11}, \ldots, e_{r r} \in S \otimes_{V} \widehat{V} \subseteq$ $A_{f} \otimes_{V} \widehat{V}$. Therefore

$$
\hat{A}:=A_{f} \otimes_{V} \widehat{V} \cong M_{r}\left(\Delta_{i j}\right),
$$

where $\Delta_{i j}=e_{i i} \hat{A} e_{j j}$. We readily see that

$$
\Delta_{i j}=\sum_{h \in G^{Z}} S_{i} \hat{x}_{h g_{i} g_{j}^{-1}} \text { and } \Delta_{i i}=\sum_{h \in G^{Z}} S_{i} \hat{x}_{h} .
$$

(Here, $\hat{x}_{g}$ is the image of $x_{g}$ under the natural embedding of $K(X ; \sigma)$ into $K(X ; \sigma) \otimes_{F\left(X^{n}\right)} \widehat{F\left(X^{n}\right)}$.)

For the moment, assume that $G^{Z} \subseteq H$. Let $v_{i}$ be a valuation on $K_{i}$ corresponding to $S_{i}$. Then by [3, Lemma 3.5] we have, for all $h \in G^{Z}, v_{i}\left(\hat{f}_{i}\left(h, g_{i} g_{j}^{-1}\right)\right)=$ $v_{i}\left(\hat{f}_{i}\left(g_{i} g_{j}^{-1}, h\right)\right)=0$. Therefore $\Delta_{i j}=\Delta_{i i} \hat{x}_{g_{i} g_{j}^{-1}}=\hat{x}_{g_{i} g_{j}^{-1}} \Delta_{j j}$.

Observe that $\Delta_{i i}=A_{\hat{f}_{i}}:=\sum_{\sigma^{j} \in G^{Z}} S_{i} \hat{x}_{\sigma^{j}}$. Since $G^{Z} \subseteq H$ and $S_{i}$ is unramified over $\hat{V}, \Delta_{i i} \cong M_{s}(\Delta)$, where $\Delta$ is the invariant valuation ring with center $\widehat{V}$ of the division algebra part of $K(X ; \sigma) \otimes_{F\left(X^{n}\right)} \widehat{F\left(X^{n}\right)}$, and $s$ is some fixed positive integer independent of $i$. Let $e_{i i}=e_{i_{1} i_{1}}+e_{i_{2} i_{2}}+\cdots+e_{i_{s} i_{s}}$ be the decomposition of $e_{i i} \in \Delta_{i i}$ into primitive orthogonal idempotents corresponding to the standard

\footnotetext{
$2 \hat{f}_{1}$ and $\hat{f}_{2}$ are not to be confused with the cocycles $f_{1}$ and $f_{2}$ defined in $\S 1$.
} 
orthogonal idempotents of $M_{s}(\Delta)$. Since $\widehat{V}$ is a DVR, Lemma 3.1 now shows that $\hat{A}$ is hereditary if and only if

$$
e_{i_{l} i_{l}} \hat{A} e_{j_{l^{\prime}} j_{l^{\prime}}} \cdot e_{j_{l^{\prime}} j_{l^{\prime}}} \hat{A} e_{i_{l} i_{l}} \supseteq e_{i_{l} i_{l}} J\left(\Delta_{i i}\right) e_{i_{l} i_{l}}
$$

for all $1 \leq i, j \leq r, 1 \leq l, l^{\prime} \leq s$. But $e_{i_{l} i_{l}} \hat{A} e_{j_{l^{\prime}} j_{l^{\prime}}} \cdot e_{j_{l^{\prime}} j_{l^{\prime}}} \hat{A} e_{i_{l} i_{l}}=e_{i_{l} i_{l}} e_{i i} \hat{A} e_{j j} e_{j_{l^{\prime}} j_{l^{\prime}}}$. $e_{j_{l^{\prime}} j_{l^{\prime}}} e_{j j} \hat{A} e_{i i} e_{i_{l} i_{l}}=e_{i_{l} i_{l}} \Delta_{i j} e_{j_{l^{\prime}} j_{l^{\prime}}} \Delta_{j i} e_{i_{l} i_{l}}=e_{i_{l} i_{l}} \hat{x}_{g_{i} g_{j}^{-1}}\left(\Delta_{j j} e_{j_{l^{\prime}} j_{l^{\prime}}} \Delta_{j j}\right) \hat{x}_{g_{j} g_{i}^{-1}} e_{i_{l} i_{l}}=$ $e_{i_{l} i_{l}} \hat{x}_{g_{i} g_{j}^{-1}} \Delta_{j j} \hat{x}_{g_{j} g_{i}^{-1}} e_{i_{l} i_{l}}=e_{i_{l} i_{l}} \Delta_{i i} \hat{x}_{g_{i} g_{j}^{-1}} \hat{x}_{g_{j} g_{i}^{-1}} e_{i_{l} i_{l}}=e_{i_{l} i_{l}} \Delta_{i i} f\left(g_{i} g_{j}^{-1}, g_{j} g_{i}^{-1}\right) e_{i_{l} i_{l}}=$ $e_{i_{l} i_{l}} \Delta_{i i}\left(e_{i i} f\left(g_{i} g_{j}^{-1}, g_{j} g_{i}^{-1}\right) e_{i i}\right) e_{i_{l} i_{l}}$.

Thus $\hat{A}$ is hereditary if and only if $f\left(g_{i} g_{j}^{-1}, g_{j} g_{i}^{-1}\right) \notin\left(M_{i}\right)^{2}$ for all $i, j$.

We now drop the assumption that $G^{Z} \subseteq H$. We will continue to assume that $X^{n} \in U(V)$ and so all maximal $V$-orders in $K(X ; \sigma)$ are Azumaya algebras, which implies that $\Delta$ is an Azumaya algebra over $\widehat{V}$. We know that $A$ is hereditary if and only if $\hat{A}$ is hereditary [5, Theorem 40.5].

Now suppose $A$ is hereditary. Then $\hat{A}$, hence $A_{\hat{f}_{i}}=e_{i i} \hat{A} e_{i i}$, a crossed-product in the sense of Haile, since $S_{i}$ is unramified over $\widehat{V}$, is hereditary. Each $A_{\hat{f}_{i}}$ is primary since $S_{i}$ is a valuation ring of $K_{i}$, and hence $A_{\hat{f}_{i}}$ is a maximal $\widehat{V}$-order in $Q_{\hat{f}_{i}}$ and so $A_{\hat{f}_{i}} \cong M_{s}(\Delta)$. We conclude that each $A_{\hat{f}_{i}}$ is Azumaya over $\widehat{V}$ and thus the subgroup of $G^{Z}$ associated to $\hat{f}_{i}$ is the whole $G^{Z}$ by the sublemma. Since $f\left(\sigma^{j}, \sigma^{-j}\right) \notin M_{i}$ for all $\sigma^{j} \in G^{Z}$ and all $i$, we conclude that $G^{Z} \subseteq H$.

So when $A_{f}$ is hereditary, then $G^{Z} \subseteq H$ and $f\left(g_{i} g_{j}^{-1}, g_{j} g_{i}^{-1}\right) \notin\left(M_{i}\right)^{2}$ for all $i, j$. Thus $A_{f}$ is hereditary if and only if $G^{Z} \subseteq H$ and $f\left(g_{i} g_{j}^{-1}, g_{j} g_{i}^{-1}\right) \notin\left(M_{i}\right)^{2}=\left(M^{2}\right)^{g_{i}}$ for all $i, j$.

When $V$ decomposes in $K\left(X^{n}\right)$ then by Theorem 2.3 there are infinitely many hereditary orders in $K(X ; \sigma)$ of the form $A_{f}$; hence there are infinitely many cohomology classes (over $S$ ) of such an $f$.

We can now state our main results:

Theorem 3.2. Let $A_{f}$ be a $V$-order as described above.

(1) Suppose $V$ is indecomposed in $K\left(X^{n}\right)$ and $X^{n}$ is a unit in $V$. Then $A_{f}$ is hereditary if and only if $H=G$, i.e., the graph of $f$ is trivial. In this case, $A_{f}$ is Azumaya over $V$, and it is the unique hereditary $V$-order of $K(X ; \sigma)$ containing $K$. We have $f \sim_{S} f_{1} \sim_{S} f_{2}$.

(2) Suppose $V$ is indecomposed in $K\left(X^{n}\right)$ and $X^{n} \in V \backslash U(V)$ (respectively $\left.X^{-n} \in V \backslash U(V)\right)$. Then $A_{f}$ is hereditary if and only if $H=\{1\}, f\left(\sigma, \sigma^{-1}\right)$ $\in J(S) \backslash J(S)^{2}$ (respectively $\left.f\left(\sigma^{-1}, \sigma\right) \in J(S) \backslash J(S)^{2}\right)$, and the graph of $f$ is

$$
\begin{gathered}
H \leq \sigma H \leq \sigma^{2} H \leq \cdots \leq \sigma^{n-1} H \\
\text { (respectively } \left.H \leq \sigma^{-1} H \leq \sigma^{-2} H \leq \cdots \leq \sigma^{1-n} H\right) .
\end{gathered}
$$

In this case, $A_{f}$ is an invariant valuation ring of $K(X ; \sigma)$, and so it is the unique hereditary $V$-order in $K(X ; \sigma)$ and hence is maximal. It is not Azumaya over $V$. We have $f \sim_{S} f_{1}$ (respectively $f \sim_{S} f_{2}$ ).

(3) Suppose $V$ decomposes in $K\left(X^{n}\right)$. Then $A_{f}$ is hereditary if and only if, for every maximal ideal $M$ of $S$ with decomposition group $G^{Z}$, we have 
$G^{Z} \subseteq H$ and there exist coset representatives $g_{1}, g_{2}, \ldots, g_{r}$ of $G^{Z}$ in $G$ such that $f\left(g_{i} g_{j}^{-1}, g_{j} g_{i}^{-1}\right) \notin\left(M^{2}\right)^{g_{i}}$ for all $i, j$.

There are infinitely many cohomology classes (over $S$ ) of such $f$ in this case.

We end by giving an example that illustrates some of the main results of this paper. For the background material and definitions of any undefined terms, the reader is referred to [5].

Example 3.3. Let $F=\mathbb{Q}, K=\mathbb{Q}(i)$, and $\sigma$ be the complex conjugation on $K$. Let $V=F\left[X^{2}\right]_{\left(X^{4}+1\right)}$. Then $X^{2} \in U(V)$. Since $X^{4}+1=\left(X^{2}-i\right)\left(X^{2}+i\right)$ in $K\left[X^{2}\right], B:=A_{f_{1}}$ and $B^{\prime}:=\left(x^{2}+i\right) B\left(X^{2}+i\right)^{-1}$ are distinct maximal $V$-orders in $K(X ; \sigma)$ containing $K$, and each one is an Azumaya algebra. Since $\left(X^{4}+1\right) B=$ $\left[B\left(X^{2}-i\right)\right] \cdot\left[\left(X^{2}+i\right) B\right]$ is a factorization of $J(B)$ into integral ideals $B\left(X^{2}-i\right)$ and $\left(X^{2}+i\right) B, A:=O_{l}\left(B\left(X^{2}-i\right)\right) \cap O_{l}\left(\left(X^{2}+i\right) B\right)=B \cap B^{\prime}$ is a hereditary $V$-order in $K(X ; \sigma)$.

In terms of crossed-product orders, note that $A=S \oplus S\left(X^{2}+i\right) X$; therefore $A=A_{f}$, where $f(1,1)=f(1, \sigma)=f(\sigma, 1)=1$ and $f(\sigma, \sigma)=\left(X^{4}+1\right) X^{2}$. Starting with such an $A_{f}$, we have $\{1\}=G^{Z}=H$ and $f(\sigma, \sigma) \notin\left(X^{2}+i\right)^{2} S,\left(X^{2}-i\right)^{2} S$, and so by Theorem 3.2 , we again see that $A_{f}$ must be hereditary.

Now let $B^{\prime \prime}=\left(x^{2}+i\right) B^{\prime}\left(X^{2}+i\right)^{-1}$ and $A^{\prime}=B \cap B^{\prime} \cap B^{\prime \prime}$. Since $V$ decomposes in $K\left(X^{2}\right), B$ is not an invariant valuation ring of $K(X ; \sigma)$; therefore the completion of $K(X ; \sigma), K(X ; \sigma) \otimes_{F\left(X^{2}\right)} \widehat{F\left(X^{2}\right)}$, is a split algebra of degree 2 . It follows that any hereditary $V$-order in $K(X ; \sigma)$ has type at most 2 , and therefore $A^{\prime}$, which is an intersection of three distinct maximal $V$-orders, cannot be hereditary.

In terms of crossed-product orders, observe that $A^{\prime}=S \oplus S\left(X^{2}+i\right)^{2} X$; therefore $A^{\prime}=A_{g}$, where $g(1,1)=g(1, \sigma)=g(\sigma, 1)=1$ and $g(\sigma, \sigma)=\left(X^{4}+1\right)^{2} X^{2}$. If we started with such an $A_{g}$, we have $g(\sigma, \sigma) \in\left(X^{2}+i\right)^{2} S,\left(X^{2}-i\right)^{2} S$, and so by Theorem 3.2, we again see that $A_{g}$ cannot be hereditary.

\section{ACKNOWLEDGEMENT}

The author thanks the referee for useful comments.

\section{REFERENCES}

1. P. M. Cohn, Algebra, Vol. II, John Wiley \& Sons, New York, 1977. MR0530404 (58:26625)

2. J. Gráter, Prime PI-rings in which finitely generated right ideals are principal, Forum Math. 4 (1992), 447-463. MR.1176882 (93i:16026)

3. D. E. Haile, Crossed-product orders over discrete valuation rings, J. Algebra 105 (1987), 116148. MR871749 (88b:16013)

4. J. S. Kauta, Integral semihereditary orders, extremality, and Henselization, J. Algebra 189 (1997), 226-252. MR1438175 (98d:16030)

5. I. Reiner, Maximal Orders, Academic Press, London, 1975. MR0393100 (52:13910)

6. A. R. Wadsworth, Dubrovin valuation rings and Henselization, Math. Ann. 283 (1989), 301328. MR $980600(90 f: 16009)$

Department of Mathematics, Faculty of Science, Universiti Brunei Darussalam, BanDAR Seri Begawan, BE1410, Brunei

E-mail address: john.kauta@ubd.edu.bn 